\title{
Epidermal growth factor receptor tyrosine kinase inhibitors for the treatment of non-small-cell lung cancer: results and open issues
}

Received: 6 June 2006 / Accepted in revised form: 31 August 2006 / Published online: 31 March 2007

\begin{abstract}
The medical treatment of non-small-cell lung cancer (NSCLC) has progressively changed since the introduction of "targeted therapy". The development of one of these molecular drug categories, e.g., the epidermal growth factor receptor (EGFR) tyrosine-kinase (TK) selective inhibitors, such as the orally active gefitinib and erlotinib, offers an interesting new opportunity. The clinical response rates obtained with their employment in unselected patient populations only account for approximately $10 \%$. Because of this, over the last two years numerous studies have been performed in order to identify the patient subsets that could better benefit from these agents. Not only patient characteristics and clinical-pathological features, such as never-smoking status, female gender, East Asian origin, adenocarcinoma histology, bronchioloalveolar subtype, but also molecular findings, such as somatic mutations in the EGFR gene, emerge as potentially useful prognostic and predictive factors in advanced NSCLC. Further, specifically designed clinical trials are still needed to completely clarify these and other open issues that are reviewed in this paper, in order to clarify all the interesting findings available in the clinical practice.
\end{abstract}

Keywords Targeted therapies - NSCLC • EGFR • TK inhibitors $\cdot$ Prognostic and predictive factors

\author{
K. Bencardino $\bullet$ M. Manzoni $\cdot$ S. Delfanti $\bullet$ A. Riccardi \\ M. Danova $(\varangle) \cdot$ G.R. Corazza \\ $1^{\text {st }}$ Department of Internal Medicine, Medical Oncology and \\ Gastroenterology \\ IRCCS San Matteo and University of Pavia \\ Piazzale C. Golgi 2, I-27100 Pavia, Italy \\ e-mail:m.danova@smatteo.pv.it
}

\section{Background}

Lung cancer mortality accounts for almost one third of all cancer-related deaths and non-small-cell lung cancer (NSCLC) represents the most frequent histotype [1]. The role of cytotoxic chemotherapy (CTx) is limited, and the prognosis remains unsatisfactory with 5-year survival rates of only $15 \%$ for all stages. The median survival of locally advanced and metastatic NSCLC does not exceed 18 months and 9 months respectively [2]. This is why the requirement for more effective treatments with fewer side effects has provoked the most recent interest in biological and clinical research.

The epidermal growth factor receptor (EGFR) is a tyrosine kinase (TK) receptor of the ErbB family, which includes ErbB1 (HER-1 or EGFR), ErbB2 (HER-2/neu), ErbB3 (HER-3) and ErbB4 (HER-4). EGFR, a 170-kDa membrane-bound protein, encoded by 28 exons, located on chromosome $7 \mathrm{p} 12$, as well as the other family members, have an integral kinase activity and an extracellular ligandbinding domain, a transmembrane region and a multifunctional cytoplasmatic tail. The latter has an ATP-binding site that allows the receptor autophosphorylation, which enhances an enzymatic pathway, involved in the cellular proliferation, angiogenesis and survival. The autophosphorylation is mediated by ligand binding, which causes receptor dimerisation, both as homodimer and as heterodimer with other members of EGFR family, preferentially with HER2. This process activates downstream signalling pathways, including the Ras/Raf/Mitogen-activated protein kinase (MAPK) pathway and the phosphatidylinositol 3-kinase (PI3K)-Akt pathway, linked to cell proliferation, transformation and survival (Fig. 1).

EGFR is over-expressed in $40 \%-80 \%$ of NSCLC patients, suggesting that the activation of the EGFR signal pathway represents one of the essential mechanisms of tumorigenesis. This is correlated with disease progression, lower response to standard therapy, development of resistance to cytotoxic drugs and overall poor survival $[3,4]$. 


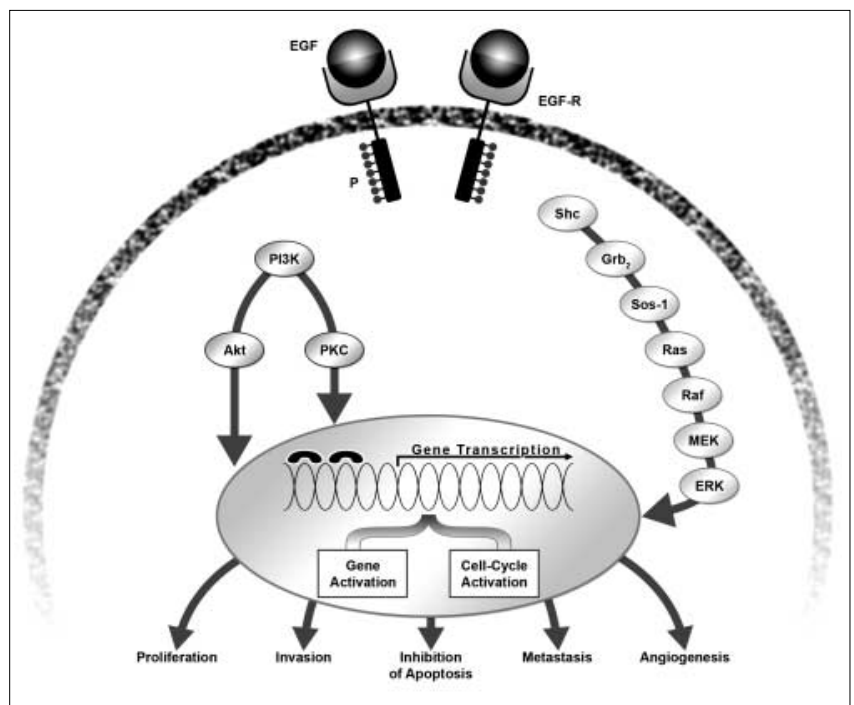

Fig. 1 EGF receptor-signal transduction pathway

The identification of small molecules able to inhibit the TK activity of EGFR, by competing with ATP for the ATP-binding site, stopping tumour cell growth by preventing the activation of EGFR, represents a new form of anticancer treatment, aimed at the blockage of a special target. Currently two of these agents have been approved by the US Food and Drugs Administration (FDA): the orally active gefitinib (Fig. 2) and erlotinib (Fig. 3).

After a long series of preclinical studies, these molecules were first clinically tested in CT-refractory patients (Table 1). Two phase II studies [5, 6] were conducted in order to test the efficacy of gefitinib at two different doses: the IDEAL-1 shows $18 \%$ partial response (PR), median survival of 8 months in Japanese and European patients who received one or two prior platinum-based CTx. The IDEAL2 shows $11 \%$ PR, median survival of 7 months in American patients who had previously received two or more regimens containing cisplatin or carboplatin and docetaxel. Disease control (objective response and stable disease) was achieved in approximately $50 \%$ of the patients in both trials, together with symptom improvement.

Two subsequent randomised phase III trials [7, 8] were conducted in previously untreated patients; a standard platinum-based CTx was employed, with or without gefitinib at two doses (INTACT-1, cisplatin and gemcitabine \pm gefitinib; INTACT-2, carboplatin and paclitaxel \pm gefitinib). The results of both studies do not show any differences in response rates, time to progression and survival with the addition of the targeted therapy to CTx.

Similar studies were conducted in order to evaluate the efficacy of erlotinib at the dose of $150 \mathrm{mg}$ : this molecule was administered in previously treated patients in a phase II trial [9], producing an objective response rate of $12 \%$ and a median survival of 8 months. Two subsequent phase III trials $[10,11]$ randomised patients in advanced stage to receive as first-line treatment a platinum-based CTx, with or without erlotinib (TALENT, cisplatin and gemc-

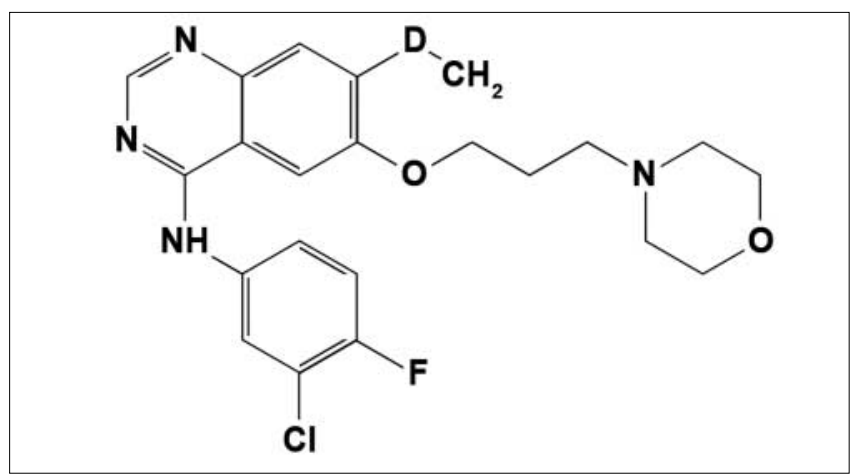

Fig. 2 Gefitinib

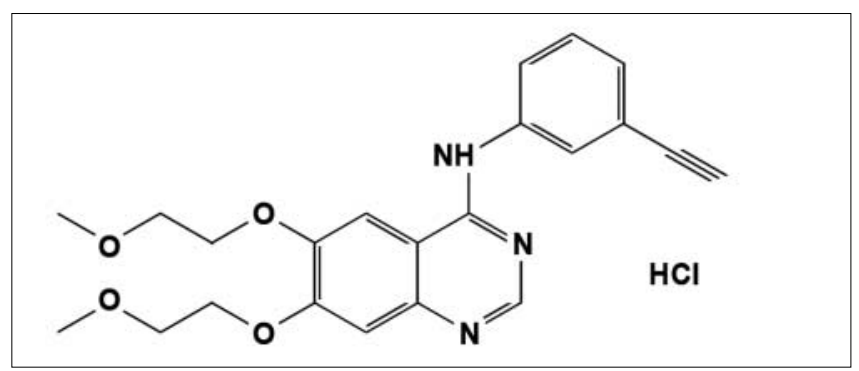

Fig. 3 Erlotinib

itabine \pm erlotinib; TRIBUTE, carboplatin and paclitaxel+erlotinib). Both studies fail to demonstrate significant clinical benefits in the experimental arm.

Two recent phase III studies focused on the efficacy of EGFR TK inhibitors in comparison with placebo. In the Canadian trial (BR. 21) patients were randomised to receive erlotinib or placebo after first or second-line CTx [12]. Statistically significant differences were observed for overall survival and progression-free survival. A similar study (ISEL) randomly assigned patients to receive gefitinib or placebo, but it failed to show any advantage in sur-

Table 1 Drug-related non-haematological toxicities by worst common toxicity criteria (CTC) grade

\begin{tabular}{lll}
\hline Adverse event & $\begin{array}{l}\text { CTC grade } \\
1-2\end{array}$ & $\begin{array}{l}\text { CTC grade } \\
3-4\end{array}$ \\
\hline $\begin{array}{l}\text { Gefitinib, } 250 \mathrm{mg} / \text { day, } n=102 \\
\text { Diarrhoea }\end{array}$ & \\
Rash & $47 \%$ & $1 \%$ \\
Acne & $43 \%$ & - \\
Dry skin & $25 \%$ & - \\
Nausea & $13 \%$ & - \\
Vomiting & $12 \%$ & $1 \%$ \\
& $11 \%$ & $1 \%$ \\
Erlotinib, $150 \mathrm{mg} /$ day, $n=485$ & & \\
Rash & $67 \%$ & $8 \%$ \\
Diarrhoea & $48 \%$ & $6 \%$ \\
Anorexia & $43 \%$ & $9 \%$ \\
Fatigue & $34 \%$ & $18 \%$ \\
Dyspnoea & $13 \%$ & $28 \%$ \\
Nausea & $30 \%$ & $3 \%$ \\
Vomiting & $21 \%$ & $2 \%$ \\
\hline
\end{tabular}


vival [13]. This data led to consideration of a possible difference between these two molecules, although the results of phase III studies differently designed could also be taken into consideration.

Safety evaluation has been primarily based on the results of trials in which patients received gefitinib or erlotinib monotherapy. From all these trials, the toxicity profile for both drugs is favourable.

No significant myelosuppression is observed. The most common non-haematological toxicities by worst common toxicity criteria grade reported at the recommended doses of gefitinib and erlotinib are shown in Table 2. They are diarrhoea, rash, dry skin, nausea and vomiting. Adverse events reported to a lesser extent were pruritus, anorexia, asthenia, weight loss, peripheral oedema, amblyopia, dyspnoea, conjunctivitis, vesiculobullous rash and mouth ulceration. Liver function test abnormalities have occasionally been observed. Cases of interstitial lung disease have been reported in patients receiving gefitinib and erlotinib with an overall incidence from all studies of approximately $1 \%$ and $0.6 \%$, respectively [14].

In this paper we have reviewed the current knowledge and pointed out the future directions of the clinical utilisation of gefitinib and erlotinib in NSCLC.

\section{Prognostic and predictive factors}

\section{Clinical and pathological features}

The overall unexpected low percentage of clinical response has been investigated in order to identify clinico- biological characteristics potentially related to a selective activity of these agents in patient subgroups.

Analysis of data from the principal trials show that response to TK inhibitors does not correlate with EGFR overexpression, but instead, well defined patient and tumour characteristics, such as female gender, non-smoking status, East Asian ethnicity, adenocarcinoma histology and bronchioloalveolar subtype, represented the features most likely associated with treatment response.

In the IDEAL 1 trial, the response rate is higher in Japanese patients $(27.5 \%$ vs. $10.4 \%, p=0.0023)$, is 2.5 times higher in women than in men and 3.5 times higher in adenocarcinoma than in other histologies [5]. In the IDEAL 2 trial, the response rate is $19 \%$ in the female population and $3 \%$ in the male, and $13 \%$ for adenocarcinoma $v s$. other histologies [6]. The Expanded Assess Program conducted at the Memorial Sloan-Kettering Cancer Center confirms better responses in women $(19 \%$ vs. $8 \%, p=0.14)$ and in adenocarcinoma (19\% vs. $0 \%, p=0.004)$, particularly in bronchioloalveolar carcinoma ( $38 \%$ vs. $14 \%, p=0.001$ ), and finds a significantly higher response rate in never-smoker patients $v s$. former or current smokers (36\% vs. 8\%, $p=0.001)$ [15].

Several smaller trials confirm the above-mentioned results, and contribute to better define the characteristics of the patient populations more responsive to this form of anticancer treatment [16].

\section{EGFR mutations}

Molecular mechanisms underlying TK inhibitor sensitivity have been recently identified, with the majority of high-

Table 2 Clinical response rate and median survival obtained with gefitinib and erlotinib in NSCLC treatment, investigated in phase II and III clinical trials

\begin{tabular}{|c|c|c|c|c|c|}
\hline Trial, year & Phase & Treatment & $\begin{array}{l}\text { Number } \\
\text { of pts }\end{array}$ & $\begin{array}{l}\text { Response rate } \\
(\%)\end{array}$ & $\begin{array}{l}\text { Median survival } \\
\text { (months) }\end{array}$ \\
\hline IDEAL 1, 2003 [5] & II & Gefitinib (250 or $500 \mathrm{mg} *$ ) & 210 & 18 & 8 \\
\hline IDEAL 2, 2003 [6] & II & Gefitinib ( 250 or $500 \mathrm{mg} *$ ) & 216 & 11 & 7 \\
\hline \multirow[t]{2}{*}{ INTACT 1, 2004 [7] } & III & $\mathrm{G} / \mathrm{C}+$ gefitinib (250 or $500 \mathrm{mg} *$ ) & 730 & 50 & 10 \\
\hline & & vs. G/C+placebo & 363 & 45 & 11 \\
\hline \multirow[t]{2}{*}{ INTACT 2, 2004 [8] } & III & $\mathrm{C} / \mathrm{P}+$ gefitinib $(250$ or $500 \mathrm{mg} *)$ & 692 & 30 & 9 \\
\hline & & vs. $\mathrm{C} / \mathrm{P}+$ placebo & 345 & 29 & 10 \\
\hline \multirow[t]{2}{*}{ TALENT , 2004 [10] } & III & G/C+erlotinib (150 mg) & 586 & 31 & 10 \\
\hline & & vs. $\mathrm{G} / \mathrm{C}+$ placebo & 586 & 30 & 10 \\
\hline \multirow[t]{2}{*}{ TRIBUTE, 2005 [11] } & III & $\mathrm{C} / \mathrm{P}+$ erlotinib $(150 \mathrm{mg})$ & 539 & 21 & 11 \\
\hline & & vs. $\mathrm{C} / \mathrm{P}+$ placebo & 540 & 19 & 10 \\
\hline \multirow[t]{2}{*}{ BR.21, 2005 [12] } & III & Erlotinib (150 mg) & 488 & 9 & 7 \\
\hline & & vs. placebo & 243 & $<1$ & 5 \\
\hline \multirow[t]{2}{*}{ ISEL, 2005 [13] } & III & Gefitinib $(250 \mathrm{mg})$ & 1129 & 8 & 5 \\
\hline & & vs. placebo & 563 & 1 & 5 \\
\hline
\end{tabular}

$G / C$, gemcitabine/cisplatin; $C / P$, carboplatin/paclitaxel; $p t s$, patients

*The trial randomisation utilised two dosages of gefitinib, 250 and $500 \mathrm{mg}$, but no differences were found in terms of efficacy 
ly responsive tumours containing somatic mutations of the EGFR gene. Several studies with different design have focused on the possible predictive value of EGFR mutations in NSCLC (Table 3).

Mutations in the EGFR TK domain are found mostly in subjects who respond to gefitinib, as reported in the first three studies conducted in 2004 [17-19]. The same mutations are more frequent in women, in adenocarcinoma, in non-smokers and in Japanese subjects, all with a statistical significance [17]. These were clustered in exons 18, 19 and 21 and were either small in-frame deletion (746-750, adjacent to K745: ELREA aminoacids) or heterozygous missense mutations (mainly L858R adjacent to the DFG motif in the $\mathrm{COOH}$-terminal lobe in the activation loop of the kinase) around the ATP binding pocket. Pooled data from these three studies show that 25 of $31(81 \%)$ patients with partial response or marked clinical improvement have an EGFR mutation, $v s$. none of 29 specimens from refractory patients $(p=0.0001)$.

Several other studies investigate the role of these mutations in the treatment of NSCLC with EGFR TK inhibitors, demonstrating a statistically significant correlation between the response to gefitinib and erlotinib and the presence of the biological data, but this correspondence is not always $100 \%$ [20-28] (Table 3). In fact objective responses are also described in patients with a wild-type EGFR, although with a lower percentage, ranging from $10 \%$ to $13 \%$ [21-23].

The probability of EGFR TK inhibitor efficacy not only increases with the presence of a mutational status, which results in the principal feature linked with response, but also correlates with female gender, non-smoker status, Asiatic origin and adenocarcinoma histology [21-28]. Other characteristics also influence tumour response, such as a greater number of prior CTx lines, or a younger age, but they are reported in only a few papers [21, 22].
Furthermore, patients with EGFR mutations survive for a longer period than those without the mutations after initiation of gefitinib treatment $(p=0.0053)$. A specific mutation (del746-750) is found to be superior to other (L858R) mutations for the prediction of response to gefitinib [23]. The same advantage in terms of time to progression (21.7 vs. 1.8 months; $p<0.001)$ and survival (30.5 vs. 6.6 months; $p<0.001)$ is demonstrated in the Korean study [25].

The treatment with gefitinib of only Japanese patients after a first recurrence shows 53\% objective responses, a median time to progression of 5 months, and a median survival of 16 months. Thirty-nine out of 66 patients had EGFR mutations, and their response rate $(82 \%$ vs. $11 \%$; $p<0.0001$ ), time to progression (12.6 vs. 1.7 months; $p<0.0001)$ and overall survival (20.4 vs. 6.9 months; $p=0.0001$ ) are higher in comparison to the wild-type patients [24]. These data once again show the higher sensitivity of the Asiatic population to EGFR-TK inhibitors.

Considering the importance of this data, the contribution of molecular alterations in EGFR to response and survival was determined in the patients treated inside the IDEAL and INTACT gefitinib trials, retrospectively. EGFR mutations were found in 14 out of 79 cases from the IDEAL studies' available tumour samples, and in 32 out of 312 cases from the INTACT trials, including amino acid substitutions, in-frame deletions clustered around the ATP binding pocket. These mutations result more frequently in adenocarcinomas than in tumours with other histologies $(17 \%$ vs. $5 \%$; $p=0.0001)$, in women than men (19\% vs. $9 \% ; p=0.006)$, in non-smokers than smokers (26\% vs. $8 \% ; p=0.0004)$ and in Asians than non-Asians (19\% vs. $11 \% ; p=0.346)$. Patients whose tumours have an EGFR mutation show a better response to gefitinib, with an overall response of $46 \%$ vs. $10 \%(p=0.005)$. Median time to progression for mutation positive cases was longer

Table 3 EGFR gene mutations and clinical response rate to EGFR TK inhibitors

\begin{tabular}{|c|c|c|c|c|c|}
\hline Author, year & Inhibitor & $\begin{array}{l}\text { Number of } \\
\text { pts }\end{array}$ & $\begin{array}{l}\text { Responder pts } \\
\text { (number) }\end{array}$ & $\begin{array}{l}\text { Number of } \\
\text { responder pts } \\
\text { with EGFR gene } \\
\text { mutation }(\%)\end{array}$ & $p$ value \\
\hline Paez et al., 2004 [17] & Gefitinib & 9 & 5 & $5(100)$ & 0.0027 \\
\hline Lynch et al., 2004 [18] & Gefitinib & 16 & 9 & $8(88)$ & 0.001 \\
\hline \multirow[t]{2}{*}{ Pao et al., 2004 [19] } & Gefitinib & 24 & 12 & $9(75)$ & 0.001 \\
\hline & Erlotinib & 36 & 10 & $8(80)$ & 0.001 \\
\hline Kondo et al., 2005 [20] & Gefitinib & 12 & 4 & $4(100)$ & NA \\
\hline Rosell et al., 2005 [21] & Gefitinib & 34 & 10 & $7(70)$ & 0.0003 \\
\hline Taron et al., 2005 [22] & Gefitinib & 68 & 22 & $16(73)$ & 0.0001 \\
\hline Mitsudomi et al., 2005 [23] & Gefitinib & 59 & 26 & $24(96)$ & 0.0001 \\
\hline Takano et al., 2005 [24] & Gefitinib & 66 & 35 & $32(91)$ & 0.0001 \\
\hline Han et al., 2005 [25] & Gefitinib & 90 & 21 & $11(52)$ & 0.001 \\
\hline Tomizawa et al., 2005 [27] & Gefitinib & 20 & 14 & $11(79)$ & 0.0022 \\
\hline Niho et al., 2005 [58] & Gefitinib & 13 & 4 & $4(100)$ & NA \\
\hline
\end{tabular}

$E G F R$, epidermal growth factor receptor; $T K$, tyrosine kinase; $p t s$, patients; $N A$, not assessed 
(116 days vs. 57 days), but no impact was detected on overall survival. Molecular analysis reveals that 13 of 18 EGFR mutation carriers (72\%) respond to chemotherapy plus gefitinib, compared to 84 of 152 mutation-negative cases $(55 \%)$, but this difference does not achieve statistical significance $(p=0.2)$ [26].

The majority of these studies were conducted testing the activity of gefitinib, but similar data were obtained with erlotinib. In particular Eberhard et al. analysed the mutational status and its impact on patients treated in the TRIBUTE trial [28]. Tumour EGFR mutations were identified in 29 of 228 subjects (12\%) and among them $17 \%$ were never smokers. The overall response rate improved in patients treated with erlotinib and CTx, who express EGFR mutations in comparison with the wild-type tumours, and both time to progression and survival are affected by EGFR mutational status. Moreover, among EGFR mutants, overall response rate is higher in the erlotinib plus CTx arm compared to CTx alone, but this does not reach statistical significance.

Finally, a recent study shows not only that EGFR mutations are statistically associated with Asian ethnicity and never-smoker status, but also that patients with EGFR exon 19 deletions have significantly longer median survival than patients with EGFR L858R mutation (34 vs. 8 months, $p=0.01$ ) after treatment with gefitinib or erlotinib [29].

These findings suggest that testing for EGFR mutations could effectively be not only of predictive but also of prognostic importance for patients with NSCLC, but the relevance of such testing in selecting patients for EGFR TK inhibitor therapy remains incompletely defined, as these data emerge from studies limited by unspecific end points and design [30].

Another unclarified point is related to the method of assessing mutations, which was different in several trials, in the absence of a standard method. To this end, recent investigations concern the validation of novel assessments, such as a dual technical approach: direct sequencing of polymerase chain reaction (PCR) products and PCR single-strand conformation (SSC) polymorphism (SSCP) analysis. The SSCP analysis results in more sensitive than direct sequencing of PCR products, and consists of a rapid and reliable method for the screening of EGFR kinase domain mutations [31]. Evaluations of newer techniques, like mutant-enriched PCR and DNA endonuclease SURVEYOR, are ongoing, resulting in valuable methods in collecting preliminary data $[32,33]$.

\section{EGFR amplifications}

The role of EGFR amplification in the relationship with NSCLC prognosis and clinical response to the treatment with small molecules like TK inhibitors is still controversial (Table 4).
Hirsch et al. first report that EGFR gene copy number correlates with EGFR protein expression, but not with prognosis [34]. Considering these data, the relationship among EGFR gene copy number, EGFR protein expression and EGFR mutations (evaluated by fluorescence in situ hybridisation (FISH) and immunohistochemistry) was further investigated in 102 NSCLC patients, treated with gefitinib. EGFR gene amplification and high protein expression are significantly associated with a better clinical response, disease control rate, time to progression and survival, while EGFR mutations correlate with clinical response and time to progression. In multivariate analysis only the EGFR amplification is significantly associated with a better survival [35]. The same statistically significant benefits in terms of response rate and time to progression are reported in a Japanese study that also observed more frequent EGFR gene amplification in patients with EGFR mutations than in patients with wildtype EGFR ( $p=0.014)$ [24].

Several other studies suggest the importance of the amplification for the activation of the EGFR signalling pathway, particularly when both gene amplification and EGFR mutation are found in the same tumour, reaching a response rate to gefitinib of $100 \%$ in the trial conducted by Taron et al. [20, 22, 24, 26]. Nevertheless the latter also showed a response rate of $45 \%$ in the patients with amplified EGFR in contrast with $89 \%$ of patients with EGFR mutations $(p=0.02)$. From these data it is difficult to determine the extent to which EGFR amplification in the absence of mutations is predictive of response [22].

Finally, the retrospective study on the tumour specimens collected in the IDEAL and the INTACT trials included not only the EGFR mutation analysis but also the EGFR gene amplification analysis by PCR. Amplification of EGFR locus was observed in 7 of 90 IDEAL cases $(8 \%)$ and in 33 of 453 INTACT cases (7\%). There is no significant increase in the prevalence of EGFR amplification in cases with clinical features that are characteristic of strong responses to gefitinib. In tumours analysed for both mutations and amplification of EGFR, 6 of 10 patients $(60 \%)$ with either genetic abnormalities had a response to gefitinib, compared with 5 of 52 patients (10\%) with neither amplification nor mutations ( $p=0.0011)$, supporting the hypothesis that genetic lesions in EGFR are critical in defining TK inhibitors susceptible subtypes of NSCLC [26].

On the contrary, Endo et al. surprisingly find that EGFR amplification does not correlate with EGFR mutation status, either with any of the clinico-pathological features or with overall survival. The authors also show a high sensitivity of TaqMan PCR to detect the mutation status [36].

On the basis of these contradictory results it is actually very difficult to give an exact definition of the role of EGFR amplification as prognostic and predictive factors, and even more difficult to clearly understand the relationship between EGFR mutations and EGFR gene amplification [37]. 
Table 4 EGFR amplification and clinical efficacy of gefitinib

\begin{tabular}{|c|c|c|c|c|c|}
\hline $\begin{array}{l}\text { Author, } \\
\text { year }\end{array}$ & $\begin{array}{l}\text { Number of } \\
\text { pts }\end{array}$ & $\begin{array}{l}\text { Number of pts } \\
\text { with EGFR } \\
\text { amplification }\end{array}$ & $\begin{array}{l}\text { Response rate }(\%) \\
\text { in pts } \\
\text { with FISH+ vs. } \\
\text { FISH- }\end{array}$ & $\begin{array}{l}\text { Time to } \\
\text { progression } \\
\text { (months) in pts } \\
\text { with FISH+ vs. } \\
\text { FISH- }\end{array}$ & $\begin{array}{l}\text { Overall survival } \\
\text { (months) in pts } \\
\text { with FISH+ vs. } \\
\text { FISH- }\end{array}$ \\
\hline Cappuzzo et al., 2005 [35] & 102 & 33 & 36 vs. $3, p=0.001$ & 9.0 vs. $2.5, p=0.001$ & 18.7 vs. $7, p=0.03$ \\
\hline Kondo et al., 2005 [20] & 12 & 2 & NA & NA & NA \\
\hline Takano et al., 2005 [24] & 66 & 29 & 72 vs. $38, p=0.005$ & 9.4 vs. $2.6, p=0.038$ & NA \\
\hline Taron et al., 2005 [22] & - & 28 & 45 (FISH - NA) & NA & NA \\
\hline Endo et al., 2005 [36] & 27 & 4 & NA & NA & No correlation \\
\hline Bell et al., 2005 [26] & 90 & 7 & 29 vs. $15, \mathrm{NA}$ & NA & NA \\
\hline
\end{tabular}

Pts, patients; EGFR, epidermal growth factor receptor; FISH, fluorescence in situ hybridisation

\section{EGFR downstream signalling}

While the mutational status seems to be important in determining the clinical response to EGFR TK-inhibitors, recent evidence suggests that genes implicated in the downstream of EGFR signalling are related not only to cancer pathogenesis but also to the clinical response to these molecular drugs.

The EGFR-dependent activation of the Ras/Raf/MAPK and PI3/Akt pathways may be involved in the sensitivity to EGFR TK inhibitors because of their role in cell proliferation and survival.

In this field, the presence of Akt in its active phosphorylated status ( $\mathrm{p}$-Akt) is associated not only with a better response to gefitinib $(p=0.003)$, disease control rate $(p<0.001)$ and time to progression $(p=0.004)$, but also with female gender $(p<0.001)$, never-smoking status $(p=0.004)$ and bronchioloalveolar carcinoma histology $(p=0.034)$. No correlation is found with p-MAPK [38].

On the contrary, no significant correlation between EGFR mutation and expression of p-Akt or p-Erk emerges in another study [25].

ErbB signalling pathways also include downstream GTPases encoded by Ras genes. K-ras mutations occur in $10 \%-30 \%$ of NSCLC cases, especially in codons 12 and 13 encoded by exon 2 , showing a strong association with smoking history and with poor prognosis [39]. Recent studies demonstrate that EGFR and K-ras mutations are mutually exclusive [40]. In fact, when the EGFR signal pathway is activated by the genetic alteration of EGFR, the mutation of Ras may not be necessary for the signal transduction, as Ras is also one of the downstream molecules in this pathway [20, 27]. The Italian investigation into the relationship between EGFR and K-ras mutations in 860 NSCLC patients reports this data: all of the tumours affected by mutated EGFR are found to be negative for K-ras mutations, whereas tumours negative for EGFR mutations show a K-ras mutation in $32 \%$ of cases $(p=0.000001)$ [31].
In the TRIBUTE trial, K-ras mutations are detected in $21 \%$ of tumours, and are associated with significantly decreased time to progression and survival in patients treated with erlotinib and chemotherapy [28]. Mutations in K-ras are found more frequently in patients who develop disease progression with either gefitinib or erlotinib [41]. Braf mutation is uncommon both in Caucasian and in Japanese NSCLC patients [42, 43], and at the moment no data is available on its role.

From these studies in particular, a molecular aetiologyand pathogenesis-related difference emerges: NSCLC arising in smokers seem to have a dissimilar spectrum of molecular alterations than those seen in non-smokers, as the basis of their opposite prognosis and responsiveness to EGFR TK inhibitors. In fact the presence of a K-ras mutation, very frequent in smokers, could likely constitute a useful marker for selecting those patients who will not benefit from anti-EGFR therapy.

The relationship between somatic mutations of EGFR and K-ras genes and DNA methylation of tumour suppressor genes has been recently investigated, and a specific interaction of genetic and epigenetic changes in the tumorigenesis of NSCLC is identified [44]. Nowadays the microarray analysis could represent a potentially useful approach to better define the complexity of NSCLC carcinogenesis, including the molecular pathways that could be associated with smoking habits and gender [45].

Finally, abnormal PI3K/Akt and Ras/Erk pathways seem to be correlated with tumour insensitivity to receptor blockade, and considering this information, the combination treatment of TK inhibitors and inhibitors of the PI3K/Akt and Ras/Erk pathways may provide a successful strategy [46].

\section{Other members of the EGFR family}

Because of the complex crosstalk among the EGFR family members, the TK inhibitor sensitivity could be related 
not only to the EGFR presence, but also to the influence of HER2 and HER3.

Preclinical data indicate that HER2, a member of the EGFR family, could enhance TK inhibitor sensitivity. EGFR family members, in fact, exist as monomers and the monomeric receptors dimerise and become functionally active after binding to the appropriate ligand; HER2 represents the preferred EGFR partner.

Independent of the method for EGFR assessment, increased copy numbers of the HER2 gene enhance sensitivity to gefitinib in patients with EGFR-positive tumours, while mutations in the TK domain of the HER2 gene seem to be infrequent and not clinically relevant. Patients with HER2 high copy number (22.8\%), detected by FISH, show significantly better objective responses, disease control rate and time to progression compared with patients with HER2 FISH-negative. Also, HER2 protein expression, tested by immunohistochemistry, shows positive results in only $7 \%$ of patients, and no HER2 mutations in exon 20 are found. HER2 gene gain is significantly associated with EGFR gene gain $(p=0.004)$ and with EGFR gene mutations $(p=0.003)$. Patients with HER2 FISH-positive tumours with an increased expression of EGFR protein, gene gain or EGFR mutations have a significantly better clinical outcome than patients negative for both receptors. On the contrary, in the absence of EGFR mutations, the outcome of HER2-positive patients is the same as the outcome of patients negative for both receptors, which is the worst scenario for all the clinical end points [47].

These data suggest a strong rationale to explore the association of TK inhibitors and anti-HER2 agents, emphasising their probable synergistic effects.

The correlation between EGFR and HER2 was also studied, with controversial results. Neither mutation nor expression of EGFR and HER2 are significantly related to the prognosis. However, the number of HER2 mutated patients was too small to accurately determine prognostic association. Despite this limitation, an interesting relationship emerges: EGFR overexpression results more frequently in tumours with EGFR mutations ( $p=0.0059)$ [48].

A series of patients treated with gefitinib were evaluated for HER3 genomic gain by FISH. HER3 FISH-positive pattern is significantly associated with female gender and never smoking history, but this gene does not emerge as a marker of response or resistance to TK inhibitors.

These data suggest a possible association between the activation of these pathways and the presence of EGFR mutations [49].

\section{Acquired resistance to EGFR TK inhibitors}

A substantial proportion of NSCLC patients treated with gefitinib and erlotinib will ultimately develop a disease relapse. The mechanism of such acquired drug resistance remains practically unknown.

First, a secondary mutation in exon 20 , leading to a substitution of methionine for threonine at position 790 (T790M) in the kinase domain, is reported in 2 of 5 patients with acquired resistance to gefitinib or erlotinib [50].

An interesting case report describes the same mutation associated with gefitinib resistance [51]: a 71-year-old smoker, with lung adenocarcinoma resistant to CTx, had a complete remission after treatment with gefitinib. After 24 months of therapy, the tumour recurred. Exons 18-21 of the EGFR gene were sequenced from DNA isolated from both the original diagnostic biopsy and the specimen obtained at the relapse: while a small deletion mutation (delL747-S752) was detected in both the biopsies, the presence of a second point mutation, resulting in threonine-to-methionine amino acid change at position 790 of EGFR (T790M), was detected only in the relapse specimen. The delL747-S752 belongs to the mutations described above associated with gefitinib responsiveness, and this report confirms these data. The appearance of a second mutation represents a mechanism of resistance: in fact the authors demonstrate that the insertion of T790M into test cells renders them resistant to gefitinib in vitro. They also find that when test cells transfected with both mutations are treated with other EGFR inhibitors, such as AG1478, cetuximab, erlotinib or CL-387,785, no objective response is obtained using the first three agents, while the fourth is effective. The sensitivity of the delL747S752+T790M construct to the anilinoquinazoline CL387,785 might be explained either by its altered binding to the kinase domain or its covalent binding to EGFR. These data support clinical investigations of compounds similar to CL-387,785 in order to identify optimal treatment strategy for patients with resistance to EGFR inhibitor therapy caused by T790M or other mutations [52]. A phase I dose escalation study was conducted in Japanese patients in order to test the efficacy of EKB-569, an oral EGFR inhibitor.

Preliminary data were recently published that show that this agent has clinical activity in 2 patients with advanced NSCLC with EGFR mutations and acquired gefitinib resistance [53]. Finally, a recent retrospective analysis of IDEAL trial specimens does not find any T790M mutations in the tumours analysed [26].

Preclinical studies are ongoing not only to investigate the biological mechanisms of resistance to EGFR TK inhibitors in greater depth, but also to identify new agents potentially able to overcome these phenomena $[54,55]$.

\section{Timing of treatment with EGFR TK inhibitors}

Several case reports are published in which EGFR TK inhibitors are utilised upfront in patients with EGFR mutations, and who are not eligible for a standard cytotoxic 
CTx [56]. These reports raise the question about the possible role of these agents as first-line therapy, and clinical studies were conducted.

A phase II study of gefitinib as first-line treatment in 36 never-smoking patients was conducted in South Korea, obtaining a response rate of $69 \%$ [57].

Another recent phase II study investigated the role of gefitinib as first-line treatment in 40 eligible patients with advanced NSCLC. In case of absence of tumour reduction within 4 weeks or partial response within 8 weeks, the treatment was interrupted, shifting to a platinum-based CTx. The response rate was $30 \%$, median survival time was 13.9 months and 4 patients developed a grade 5 interstitial lung disease $(10 \%)$. The response rate was statistically correlated with adenocarcinoma histology $(p=0.0048)$, female gender $(p=0.0050)$ and non-smoking status $(p=0.0048)$. Tumour samples were available for 13 patients ( 4 partial response, 6 stable disease and 3 progressive disease): EGFR mutations were detected in 4 responder patients. The response rate of the second line treatment was $30 \%$, and it does not seem to be adversely affected by pre-treatment with gefitinib [58].

Nowadays a key question about the use of EGFR TK inhibitors in NSCLC is the rationale of their employment as first-line treatment of advanced disease as well as neoadjuvant or adjuvant treatment in those patients whose tumours have EGFR mutations. In fact, the real impact of EGFR mutations in making a therapeutic decision has not been well defined because this target included the majority but not all responders, as has been revealed by the above-mentioned studies.

To date, no conclusive data are available from large, prospective clinical trials evaluating these agents in earlier treatment setting, and the availability of such biologic drugs approved for the treatment of recurrent disease selected patients.

\section{Conclusions and open issues}

During recent years, the development of targeted anticancer therapy has become more promising than the optimisation of medical treatment with conventional anticancer agents. In NSCLC, the targeting of EGFR TK, by the small molecules gefitinib and erlotinib, has led to more options in the management of patients with advanced dis- ease. The two drugs followed the same development procedure and, in spite of the large number of studies performed to date, further research is still warranted to define the ultimate clinical role of these agents.

In the field of prognostic and predictive factors that will aid a clinically useful patient selection, it is necessary to initiate large and well designed clinical trials with EGFR TK inhibitors in different regions of the world, and to focus on patient subsets characterised by specific clinical and histopathological features.

Furthermore, our understanding of the molecular profile in a tumour that may predict clinical response remains naïve, and as the molecular heterogeneity of NSCLC becomes more apparent, it is necessary to direct the research to identify the biomarkers that will indicate which patients are most likely to experience therapeutic benefit. To this end it is essential to incorporate the EGFR mutational profile and the characterisation of the genes involved in the EGFR signalling, into specifically designed clinical trials. This also means the urgent need for a careful standardisation of laboratory methods for molecular marker characterisation.

Preclinical studies continue to be important, in particular to better define the potential role of the association between different EGFR family members as well as to elucidate the mechanisms of resistance to EGFR inhibitors (and the possible strategies to overcome it).

At the same time, efforts should be directed to a better understanding of useful strategies for associating these agents with conventional CTx, also taking into account that no exhaustive data is available on the possible effect of CTx on EGFR expression or mutations.

Future research should also involve the identification of the phase of disease in which these agents can be best employed (such as the neoadjuvant and the adjuvant setting), together with the validation of new surrogate markers of clinical response to these drugs. In fact, the effect of molecular agents on tumour cells seems to be cytostatic rather than cytotoxic so that the standard methodology utilised for the assessment of efficacy in clinical cancer research may not be the best one to test for the efficacy evaluation of the targeted therapy.

In conclusion, EGFR TK inhibitors are promising anticancer agents, but all the questions mentioned above have not been completely answered by the relatively high number of studies conducted to date (Table 5). Hence, designing spe-

Table 5 Open issues about EGFR TK inhibitors employment in the treatment of NSCLC

Identification and validation of clinical and biological prognostic/predictive factors

Definition of standard methods for molecular target characterisation

Importance of the relationship among different EGFR family members

Better comprehension of the mechanisms of resistance and their possible treatment

Role of the association with conventional chemotherapy

Utilisation as neoadjuvant, adjuvant and first-line treatment

Specifically designed clinical trials including new surrogate end points, conducted in selected patient populations 
cific clinical trials to further investigate the activity of this drug class and optimise their use in prospectively defined patient populations are critical challenges to the final success of this therapeutic approach. Only in this way, within the next few years, will we find out whether we can make a real paradigm shift in the treatment of NSCLC by translating all the basic scientific progress into clinical practice.

\section{References}

1. - (2006) Leading sites of new cancer cases and deaths - 2006 estimates. Cancer facts and figure 2006. American Cancer Society

2. Pfister DG, Johnson DH, Azzoli CG et al (2004) American Society of Clinical Oncology treatment of unresectable nonsmall-cell lung cancer guideline: update 2003. J Clin Oncol 22:330-353

3. Brabender J, Danenberg KD, Metzger R et al (2001) Epidermal growth factor receptor and HER2-neu mRNA expression in non-small cell lung cancer is correlated with survival. Clin Cancer Res 7:1850-1855

4. Nicholson RI, Gee JM, Harper ME (2001) EGFR and cancer prognosis. Eur J Cancer 37[Suppl 4]:S9-S15

5. Fukuoka M, Yano S, Giaccone G et al (2003) Multi-institutional randomized phase II trial of gefitinib for previously treated patients with advanced non-small-cell lung cancer. J Clin Oncol 21:2237-2246

6. Kris MG, Natale RB, Herbst RS et al (2003) Efficacy of gefitinib, an inhibitor of the epidermal growth factor receptor tyrosine kinase, in symptomatic patients with non-small cell lung cancer: a randomized trial. JAMA 290:2149-2158

7. Giaccone G, Herbst RS, Manegold C et al (2004) Gefitinib in combination with gemcitabine and cisplatin in advanced nonsmall cell lung cancer: a phase III trial INTACT-1. J Clin Oncol 22:777-784

8. Herbst RS, Giaccone G, Schiller JH et al (2004) Gefitinib in combination with paclitaxel and carboplatin in advanced non-small cell lung cancer: a phase III trial INTACT-2. J Clin Oncol 22:785-794

9. Perez-Soler R (2004) Phase II clinical trial data with the epidermal growth factor receptor tyrosine kinase inhibitor erlotinib (OSI-774) in non-small-cell lung cancer. Clin Lung Cancer 6[Suppl 1]:S20-S23

10. Gatzemeier U, Pluzanska A, Szczesna A et al (2004) Results of a phase III trial of erlotinib (OSI-774) combined with cisplatin and gemcitabine (GC) chemotherapy in advanced nonsmall cell lung cancer (NSCLC) [abstract]. ASCO meeting abstracts 22:617 (abstr 7010)

11. Herbst RS, Prager D, Hermann R et al (2005) TRIBUTE: a phase III trial of erlotinib hydrochloride (OSI-774) combined with carboplatin and paclitaxel chemotherapy in advanced non-small-cell lung cancer. J Clin Oncol 23:5892-5899

12. Shepherd FA, Rodrigues Pereira J, Ciuleanu T et al (2005) Erlotinib in previously treated non-small-cell lung cancer. N Engl J Med 353:123-132

13. Tamura K, Fukuoka M (2005) Gefitinib in non-small cell lung cancer. Expert Opin Pharmacother 6:985-993

14. Siegel-Lakhai WS, Beijnen JH, Schellens JH (2005) Current knowledge and future directions of the selective epidermal growth factor receptor inhibitors erlotinib (Tarceva) and gefitinib (Iressa). Oncologist 10:579-589

15. Miller VA, Kris MG, Shah N et al (2004) Bronchioloalveolar pathologic subtype and smoking history predict sensitivity to gefitinib in advanced non-small-cell lung cancer. J Clin Oncol 22:1103-1109

16. Giaccone G (2005) Epidermal growth factor receptor inhibitors in the treatment of non-small-cell lung cancer. J Clin Oncol 23:3235-3242

17. Paez JG, Janne PA, Lee JC et al (2004) EGFR mutations in lung cancer: correlation with clinical response to gefitinib therapy. Science 304:1497-1500

18. Lynch TJ, Bell DW, Sordella R et al (2004) Activating mutations in the epidermal growth factor receptor underlying responsiveness of non-small-cell lung cancer to gefitinib. $\mathrm{N}$ Engl J Med 350:2129-2139

19. Pao W, Miller V, Zakowski M et al (2004) EGF receptor gene mutations are common in lung cancers from «never smokers» and are associated with sensitivity of tumors to gefitinib and erlotinib. Proc Natl Acad Sci USA 101:13306-13311

20. Kondo M, Yokoyama T, Fukui T et al (2005) Mutations of epidermal growth factor receptor of non-small cell lung cancer were associated with sensitivity to gefitinib in recurrence after surgery. Lung Cancer 50:385-391

21. Rosell R, Ichinose $\mathrm{Y}$, Taron M et al (2005) Mutations in the tyrosine kinase domain of the EGFR gene associated with gefitinib response in non-small-cell lung cancer. Lung Cancer 50:25-33

22. Taron M, Ichinose $\mathrm{Y}$, Rosell R et al (2005) Activating mutations in the tyrosine kinase domain of the epidermal growth factor receptor are associated with improved survival in gefitinib-treated chemorefractory lung adenocarcinomas. Clin Cancer Res 11:5878-5885

23. Mitsudomi T, Kosaka T, Endoh H et al (2005) Mutations of the epidermal growth factor receptor gene predict prolonged survival after gefitinib treatment in patients with non-smallcell lung cancer with postoperative recurrence. J Clin Oncol 23:2513-2520

24. Takano T, Ohe Y, Sakamoto H et al (2005) Epidermal growth factor receptor gene mutations and increased copy numbers predict gefitinib sensitivity in patients with recurrent nonsmall-cell lung cancer. J Clin Oncol 23:6829-6837

25. Han S, Kim T, Hwang PG et al (2005) Predictive and prognostic impact of epidermal growth factor receptor mutation in non-small-cell lung cancer patients treated with gefitinib. J Clin Oncol 23:2493-2501

26. Bell DW, Lynch TJ, Haserlat SM et al (2005) Epidermal growth factor receptor mutations and gene amplification in non-small-cell lung cancer: molecular analysis of the IDEAL/INTACT gefitinib trials. J Clin Oncol 23:8081-8092

27. Tomizawa Y, Iijima H, Sunaga N et al (2005) Clinicopathologic significance of the mutations of the epidermal growth factor receptor gene in patients with non-smallcell lung cancer. Clin Cancer Res 11:6816-6822

28. Eberhard DA, Johnson BE, Amler LC et al (2005) Mutations in the epidermal growth factor receptor and in KRAS are predictive and prognostic indicators in patients with non-smallcell lung cancer treated with chemotherapy alone and in combination with erlotinib. J Clin Oncol 23:5900-5909

29. Riely GJ, Pao W, Pham D et al (2006) Clinical course of 
patients with non-small-cell lung cancer and epidermal growth factor receptor exon 19 and exon 21 mutations treated with gefitinib or erlotinib. Clin Cancer Res 12:839-844

30. Shigematsu H, Gazdar AF (2006) Somatic mutations of epidermal growth factor receptor signaling pathway in lung cancers. Int J Cancer 118:257-262

31. Marchetti A, Martella C, Felicioni L et al (2005) EGFR mutations in non-small-cell lung cancer: analysis of a large series of cases and development of a rapid and sensitive method for diagnostic screening with potential implications on pharmacologic treatment. J Clin Oncol 23:857-865

32. Asano H, Toyooka S, Tokumo M et al (2006) Detection of EGFR gene mutation in lung cancer by mutant-enriched polymerase chain reaction assay. Clin Cancer Res 12:43-48

33. Janne PA, Borras AM, Kuang Y et al (2006) A rapid and sensitive enzymatic method for epidermal growth factor receptor mutation screening. Clin Cancer Res 12:751-758

34. Hirsch FR, Varella-Garcia M, Bunn PA Jr et al (2003) Epidermal growth factor receptor in non-small-cell lung carcinomas: correlation between gene copy number and protein expression and impact on prognosis. J Clin Oncol 21:3798-3807

35. Cappuzzo F, Hirsch FR, Rossi E et al (2005) Epidermal growth factor receptor gene and protein and gefitinib sensitivity in non-small-cell lung cancer. J Natl Cancer Inst 97:643-655

36. Endo K, Konishi A, Sasaki H et al (2005) Epidermal growth factor receptor gene mutation in non-small cell lung cancer using highly sensitive and fast TaqMan PCR assay. Lung Cancer 50:375-384

37. Johnson BE, Janne PA (2005) Selecting patients for epidermal growth factor receptor inhibitor treatment: a FISH story or a tale of mutations? J Clin Oncol 23:6813-6816

38. Cappuzzo F, Magrini E, Ceresoli GL et al (2004) Akt phosphorylation and gefitinib efficacy in patients with advanced non-small-cell lung cancer. J Natl Cancer Inst 96:1133-1141

39. Ahrendt SA, Decker PA, Alawi EA et al (2001) Cigarette smoking is strongly associated with mutation of the K-ras gene in patients with primary adenocarcinoma of the lung. Cancer 92:1525-1530

40. Shigematsu H, Lin L, Takahashi T et al (2005) Clinical and biological features associated with epidermal growth factor receptor gene mutations in lung cancers. J Natl Cancer Inst 97:339-346

41. Pao W, Wang TY, Riely GJ et al (2005) KRAS mutations and primary resistance of lung adenocarcinomas to gefitinib or erlotinib. PLoS Med 2:e17

42. Sasaki H, Kawano O, Endo K et al (2006) Uncommon V599E BRAF mutations in Japanese patients with lung cancer. J Surg Res 133:203-206

43. Brose MS, Volpe P, Feldman M et al (2002) BRAF and RAS mutations in human lung cancer and melanoma. Cancer Res 62:6997-7000

44. Toyooka S, Tokumo M, Shigematsu H et al (2006) Mutational and epigenetic evidence for independent pathways for lung adenocarcinomas arising in smokers and never smokers. Cancer Res 66:1371-1375

45. Shibata T, Uryu S, Kokubu A et al (2005) Genetic classification of lung adenocarcinoma based on array-based comparative genomic hybridization analysis: its association with clinicopathologic features. Clin Cancer Res 11:6177-6185

46. Janmaat ML, Rodriguez JA, Gallegos-Ruiz M, Kruyt FA, Giaccone G (2006) Enhanced cytotoxicity induced by gefitinib and specific inhibitors of the Ras or phosphatidyl inositol-3 kinase pathways in non-small cell lung cancer cells. Int J Cancer 118:209-214

47. Cappuzzo F, Varella-Garcia M, Shigematsu H et al (2005) Increased HER2 gene copy number is associated with response to gefitinib therapy in epidermal growth factor receptor-positive non-small cell lung cancer patients. J Clin Oncol 23:5007-5018

48. Suzuki M, Shigematsu H, Hiroshima K et al (2005) Epidermal growth factor receptor expression status in lung cancer correlates with its mutation. Hum Pathol 36:1127-1134

49. Cappuzzo F, Toschi L, Domenichini I et al (2005) HER3 genomic gain and sensitivity to gefitinib in advanced nonsmall-cell lung cancer patients. Br J Cancer 93:1334-1340

50. Pao W, Miller VA, Politi KA et al (2005) Acquired resistance of lung adenocarcinomas to gefitinib or erlotinib is associated with a second mutation in the EGFR kinase domain. PLoS Med 2:e73

51. Kobayashi S, Boggon TJ, Dayaram T et al (2005) EGFR mutation and resistance of non-small-cell lung cancer to gefitinib. N Engl J Med 352:786-792

52. Kobayashi S, Ji H, Yuza Y et al (2005) An alternative inhibitor overcomes resistance caused by a mutation of the epidermal growth factor receptor. Cancer Res 65:7096-7101

53. Yoshimura N, Kudoh S, Kimura T et al (2006) EKB-569, a new irreversible epidermal growth factor receptor tyrosine kinase inhibitor, with clinical activity in patients with nonsmall cell lung cancer with acquired resistance to gefitinib. Lung Cancer 51:363-368

54. Ando K, Ohmori T, Inoue F et al (2005) Enhancement of sensitivity of tumor necrosis factor alpha in non-small cell lung cancer cells with acquired resistance to gefitinib. Clin Cancer Res 11:8872-8879

55. Shah NP, Tran C, Lee FY et al (2004) Overriding imatinib resistance with a novel ABL kinase inhibitor. Science 305:399-401

56. Lange T, Muller-Tidow C, Serve H et al (2005) First-line systemic treatment with gefitinib in stage IV non-small cell lung cancer. Onc Rep 14:1539-1542

57. Lee DH, Han JY, Lee HG et al (2005) Gefitinib as a first-line therapy of advanced or metastatic adenocarcinoma of the lung in never-smokers. Clin Cancer Res 11:3032-3037

58. Niho S, Kubota K, Goto K et al (2006) First-line single agent treatment with gefitinib in patients with advanced non-small cell lung cancer: a phase II study. J Clin Oncol 24:64-69 